\title{
Structural Insights into the Recognition Properties of Human Ficolins
}

\author{
Virginie Garlatti Lydie Martin Monique Lacroix Evelyne Gout \\ Gérard J. Arlaud Nicole M. Thielens Christine Gaboriaud \\ Institut de Biologie Structurale JP Ebel, Grenoble, France
}

\section{Key Words}

Complement $\cdot$ Carbohydrate specificity $\cdot$ Ficolins $\cdot$ Innate immunity $\cdot$ Pattern Recognition Receptor $\cdot$ X-ray structures the atomic level. In the case of M-ficolin, a structural transition at acidic $\mathrm{pH}$ disables the binding pocket, and thus likely participates in the functional cycle of this protein.

Copyright $\odot 2009$ S. Karger AG, Basel
The mammalian immune system has evolved innate and adaptive components that cooperate to protect the host against microbial infections while maintaining integrity of the body. The innate system involves various constitutive recognition molecules able to sense both exogenous and endogenous danger signals arising from pathogens or damaged host cells. This triggers effector mechanisms aimed at limiting early infection and clearing apoptotic cells while stimulating an appropriate adaptive response (immunogenic vs. tolerogenic) [1].

Ficolins are members of the defense collagens family. They are oligomeric proteins with globular recognition domains able to sense danger signals and collagen-like stalks providing a link with immune effectors. In humans, 3 ficolins have been characterized [2-5]: L-ficolin/ P35 (or ficolin 2) and H-ficolin (or ficolin 3) are serum proteins, whereas M-ficolin (or ficolin 1) is mainly localized in secretory granules of various cell types, being detected recently in plasma at very low levels [6]. L-ficolin has been reported to bind to several strains of capsu-

\section{KARGER}

Fax +4161306 1234

E-Mail karger@karger.ch

www.karger.com
(C) 2009 S. Karger AG, Basel

$1662-811 X / 10 / 0021-0017 \$ 26.00 / 0$

Accessible online at:

www.karger.com/jin
Dr. Christine Gaboriaud

Institut de Biologie Structurale JP Ebel

41 rue Jules Horowitz

FR-38041 Grenoble Cedex 1 (France)

Tel. +33 43878 9599, Fax +3343878 5122, E-Mail christine.gaboriaud@ibs.fr 


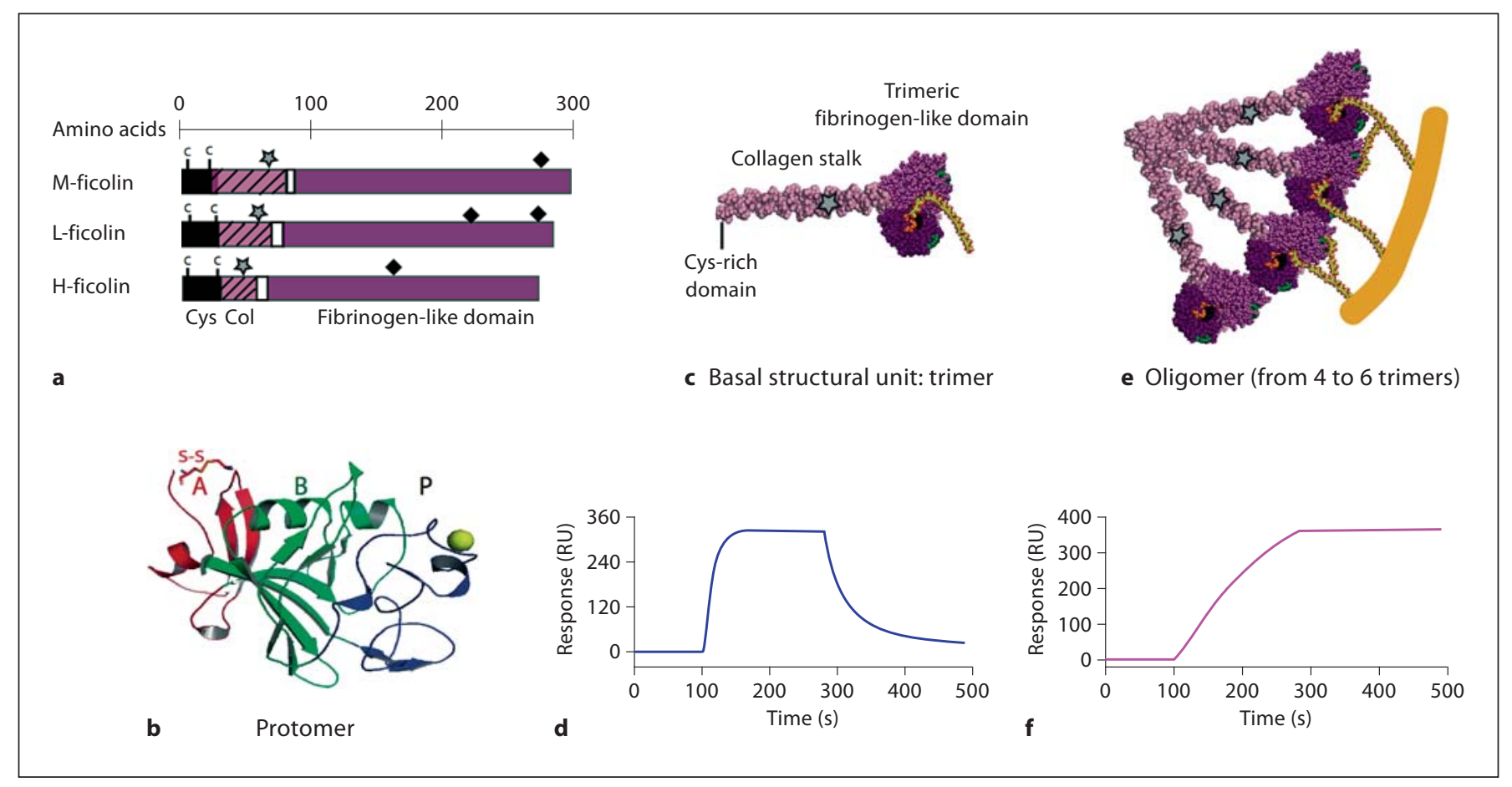

Fig. 1. Structural organization of ficolins and their multivalent recognition function. a Each mature polypeptide chain comprises a cysteine-rich $\mathrm{N}$-terminal sequence (black), a collagen-like sequence (hatched), a linker segment (white) and a fibrinogen-like sequence (purple). The N-linked oligosaccharides $(\boldsymbol{})$, the binding site for the MASPs ( $\downarrow$ ) and the $2 \mathrm{~N}$-terminal cysteine residues are indicated. $\mathbf{b}$ X-ray structure of the fibrinogen-like protomer. Domains $\mathrm{A}, \mathrm{B}$ and $\mathrm{P}$ are colored red, green and blue respectively. The N-terminal disulfide bond (S-S) is shown in red. $\mathrm{A} \mathrm{Ca}^{2+}$ ion is represented by a yellow sphere. $\mathbf{c}$ Three polypeptide chains as-

lated Gram-positive bacteria and to recognize a wide variety of ligands, such as acetylated compounds, lipoteichoic acid, 1,3- $\beta$-D-glucan, and the capsular polysaccharide of type III group B streptococci [7-10]. H-ficolin was shown to specifically bind to Aerococcus viridans and to a polysaccharide derived from this bacterium [11], and recent data provide evidence for its ability to recognize acetyl groups such as those of acetylated BSA [12]. Recombinant M-ficolin exhibits a binding specificity for acetylated carbohydrates such as GlcNAc, GalNAc and sialyl-N-acetyllactosamine $[13,14]$. Binding to Escherichia coli, the smooth type strain of Salmonella typhimurium, and Staphylococcus aureus has also been reported [15].

From a structural point of view, ficolins are assembled from basal homotrimeric subunits comprising a collagen-like triple helix and a globular recognition domain semble to form the basal structural unit. The predicted external binding site $\mathrm{S} 1$, which is observed in the case of $\mathrm{H}$ - and $\mathrm{M}$-ficolins, is shown in green. Glycans (yellow) bind to the trimeric fibrinogen-like domains (model derived from the X-ray structure of Lficolin). $\mathbf{d}$ Analysis by surface plasmon resonance spectroscopy of the binding of the L-ficolin trimeric fibrinogen-like domains to immobilized acetylated BSA. e Schematic illustration of the multivalent binding of L-ficolins oligomers on surface glycans (yellow). $\mathbf{f}$ Analysis by surface plasmon resonance spectroscopy of the binding of oligomeric L-ficolin to immobilized acetylated BSA. composed of 3 fibrinogen-like domains (fig. 1a, c). Two cysteines at the $\mathrm{N}$-terminal end of the polypeptide chains form interchain disulfide bonds that mediate assembly into higher oligomeric structures (fig. la, c, e). Like mannan-binding lectin (MBL), ficolins are able to trigger the complement cascade through activation of the associated protease MASP-2 [16, 17]. The MASP interaction site within the collagen-like regions of human MBL and ficolins has been recently mapped to a short sequence stretch centered on a conserved lysine residue (fig. la, c) $[12,18]$. Binding of the collectin receptor calreticulin competes for the same site on the collagen stalks $[12,18]$. This interaction with a receptor likely plays a role in the clearance of pathogens and cell debris. Two other MBL/ficolin-associated proteases, MASP-1 and MASP-3, have been described $[19,20]$, as well as a truncated form of MASP-2, termed MAp19 or sMAp [21, 22]. These pro- 


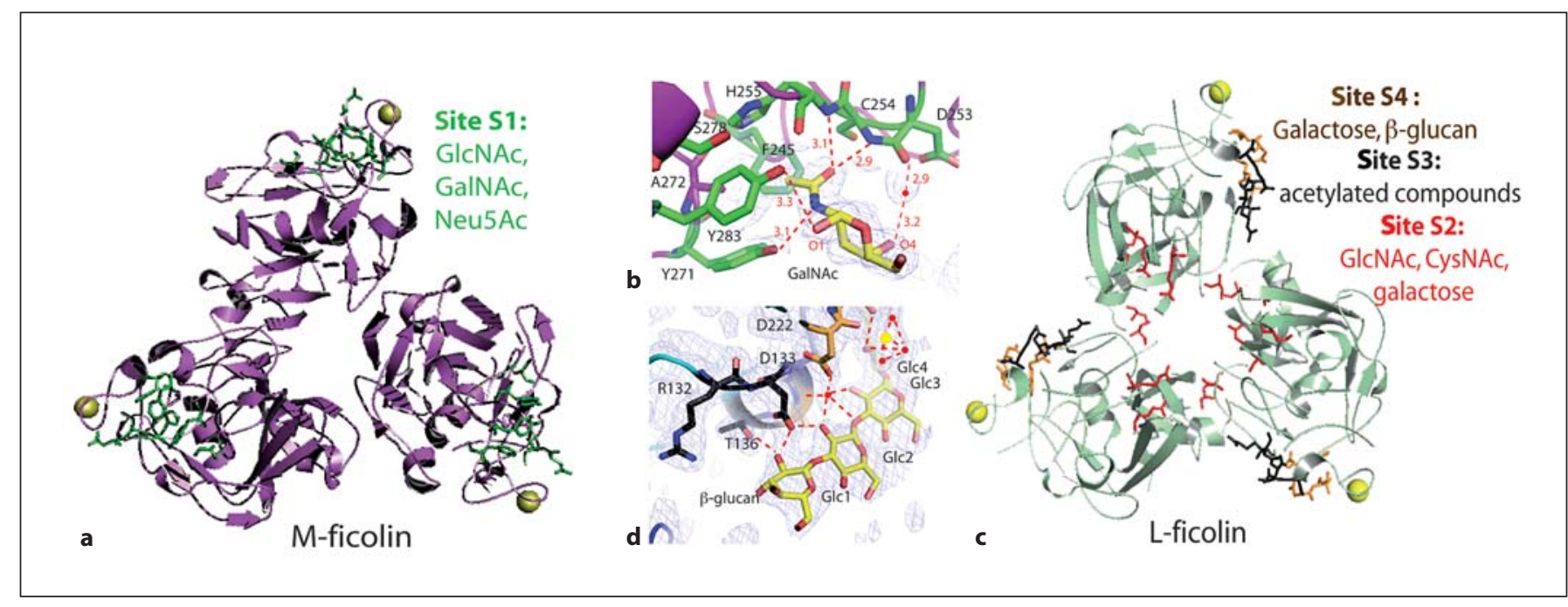

Fig. 2. The ligand binding sites in M- and L-ficolins. a An outer binding site (S1, in green) was observed for GlcNAc, GalNAc and Neu5Ac, on each protomer of the trimeric fibrinogen-like domain of M-ficolin. b Binding of GalNAc in site S1 of M-ficolin. c Three binding sites S2 (red), S3 (black) and S4 (orange) were observed in the trimeric fibrinogen-like domain of L-ficolin, which binds a wide diversity of acetylated ligands and some neutral sugars (ga- lactose, $\beta$-D-glucan). d Details of the interaction between 4 consecutive glucose units of $\beta$-D-glucan and sites S3 and S4 in L-ficolin. Polar interactions are represented by dashed red lines, with the corresponding distance in $\AA$. Water molecules are shown as red dots and an additional $\mathrm{Ca}^{2+}$ ion is represented by a small yellow sphere. teins also bind to the same site on the collagen stalks but their function remains elusive.

As described for MBL, oligomerization of ficolins through their collagen-like regions plays a crucial role in their binding to carbohydrate or acetylated patterns. As illustrated in figure $1 \mathrm{~d}-\mathrm{f}$, interaction of the isolated trimeric recognition domain of L-ficolin with immobilized acetylated BSA is characterized by a high dissociation rate whereas the full-length oligomeric protein forms a very stable complex because of its multivalent binding avidity (fig. 1f). This review will focus on the structure and recognition properties of the 3 human ficolins, as revealed by recent crystallographic analysis of their recognition domains.

\section{Conserved Overall Trimeric Structure}

The fibrinogen-like recognition domains of human L-, H- and M-ficolins have been produced recombinantly using baculovirus/insect cells or yeast expression systems, and their 3-dimensional structures have been solved by X-ray crystallography at resolutions of 1.5-1.9 $\AA$ [2325]. As expected from the trimeric nature of ficolins, these domains were found to form homotrimers in each case.
As illustrated for M- and L-ficolins (fig. 2a, c), the protomers associate as 3-lobed clover-like structures with pseudo-3-fold symmetry. The assemblies feature a small central hole and are rather open, the outer part of the protomers being separated by clefts. The N-terminal ends of the 3 protomers emerge at the base of the trimers, consistent with the fact that they are normally connected to a collagen-like triple helix in the intact proteins (fig. 1c, e).

The protomers have an ellipsoidal structure similar to that of tachylectin 5A (TL5A) [26], featuring the 3 domains $\mathrm{A}, \mathrm{B}$ and $\mathrm{P}$ originally described for this protein (fig. 1b). The N-terminal A domain contains an extra disulfide bridge not present in TL5A. The larger domain B, comprising a 7 -stranded anti-parallel $\beta$-sheet and $2 \alpha$ helices, is clamped between domains A and P. Each P domain contains a $\mathrm{Ca}^{2+}$ binding site located in the most external part of the trimers (fig. 2a, c), and coordination of the $\mathrm{Ca}^{2+}$ ion in each ficolin involves residues strictly homologous to those described in TL5A ( $\mathrm{Asp}^{233}, \mathrm{Asp}^{235}$, $\mathrm{Ser}^{237}$ and $\mathrm{Ser}^{239}$ in M-ficolin). The 3 ficolins exhibit highly similar overall protomer structures, with a root mean square deviation of $0.5 \AA$ between L- and M-ficolins, based on $211 \mathrm{C} \alpha$ atoms.

The interprotomer interfaces involve homologous residues mainly contributed by domain $\mathrm{A}$ on one side and 
domain B on the other [see 23 for more details]. These provide a conserved interaction framework consisting of hydrogen bonds, a salt bridge, and hydrophobic interactions. The total buried surface at the 3 interfaces is approximately $2,900 \AA^{2}$, a value that reflects a semi-open structure intermediate between the compact assembly seen in complement protein Clq [27] and the widely open structure of the carbohydrate recognition domain of MBL [28]. An interesting feature of L-ficolin is the subtle variability of the angle between the $\mathrm{A}, \mathrm{B}$ and $\mathrm{C}$ protomers, arising from the implication of a variable number of hydrophobic contacts. This remarkable plasticity of the interprotomer interfaces may be relevant to the recognition function of L-ficolin.

\section{Single Conserved External Binding Site in $\mathrm{H}$ - and M-Ficolins}

A single ligand binding site was identified in $\mathrm{H}$ - and M-ficolins [23-24]. It is located on the external part of the trimer, in the vicinity of the $\mathrm{Ca}^{2+}$ binding site (fig. 1c, 2a). This site is referred to as $\mathrm{S} 1$, because it is homologous to the one previously described for TL5A [26]. In M-ficolin, GlcNAc, GalNAc and Neu5Ac were bound in S1 mainly through their $\mathrm{N}$-acetyl moiety. This selective binding specificity for the $\mathrm{N}$-acetyl group was also observed in TL5A. Comparison of the different ligands bound to Mficolin and TL5A reveals a common scheme for stabilization of the $\mathrm{N}$-acetyl group (fig. 2b). The nitrogen is bound to a conserved tyrosine (Tyr271), and the oxygen is bound to 2 adjacent main chain nitrogens (contributed by Cys 254 and His255), maintained in this particular orientation through the unusual cis conformation of the cysteine. Finally, the methyl group is inserted into a conserved hydrophobic and aromatic pocket. Besides this common framework, additional interactions stabilize the diverse ligands [see 24 for more details]. In the case of $\mathrm{H}$-ficolin, the homologous S1 site has been significantly modified, altering its overall shape and thereby its fine specificity [23]. For example, only D-fucose and galactose were found to bind to $\mathrm{H}$-ficolin, but not the M-ficolin ligands [see 23 for more details].

\section{M-ficolin Is a Sensor of Sialic Acid and Subject to pH-Dependent Regulation}

M-ficolin and its rat counterpart ficolin B can bind Neu5Ac, a property which is not shared by the other ficolins. This is quite intriguing, since sialic acid is usually considered as a hallmark of 'self'. Again, this ligand is bound through its $\mathrm{N}$-acetyl group, as described above. However, some residues in the vicinity of S1 are replaced by amino acids with a shorter side chain (Gly221, Ala256), likely accounting for this uncommon property to accommodate such a large ligand [24].

The $\mathrm{S} 1$ site in $\mathrm{M}$-ficolin is completely dislocated at $\mathrm{pH}$ 5.6. The transition to acidic $\mathrm{pH}$ induces the concerted displacement of 4 surface loops, resulting in significant displacements (>10 A) of the residues defining S1; moreover, the Asp253-Cys254 bond goes from the cis-conformation required for proper ligand binding $[24,25]$ to an inactive trans-conformation. This suggests that the binding properties of $\mathrm{M}$-ficolin can be regulated by the local $\mathrm{pH}$. We have investigated the $\mathrm{X}$-ray structures of $\mathrm{H}$ - and L-ficolins at acidic $\mathrm{pH}$ and did not find any such conformational modification (data not shown). In M-ficolin, 3 histidines $\mathrm{H} 222$, H255 and $\mathrm{H} 268$ are likely to play a role in the $\mathrm{pH}$-dependent conformational transition. Indeed, these residues mediate stabilizing interactions at neutral $\mathrm{pH}$ which are lost at acidic $\mathrm{pH}$ [25], and their mutation to alanine profoundly affects GlcNAc binding [29].

\section{L-ficolin Uses a Unique Extended Recognition Surface to Sense Various Acetylated and Neutral Carbohydrates in Large Polysaccharides}

Because of its broader binding specificity, a wider range of ligands was used in the study of L-ficolin, including acetylated compounds and neutral carbohydrates [23]. However, we did not observe additional electron density corresponding to the studied ligands inside S1. One explanation for this discrepancy is that the S1 tyrosine residue that is involved in the common $\mathrm{N}$-acetyl group stabilization scheme described above, is replaced by a phenylalanine in L-ficolin. Quite unexpectedly, we observed that the ligands were mainly bound to hitherto unidentified sites that we named S2, S3 and S4 (fig. 2c). Thus, GlcNAc, CysNAc and galactose were found to bind in the highly polar $\mathrm{S} 2$ cavity, various $\mathrm{N}$-acetylated compounds bound in $S 3$, whereas the more extended $\beta-D$ glucan molecule was bound along the external sites S3 and S4. Together, these novel sites define a unique extended recognition surface.

During these studies, we have observed different levels of map quality for the extra experimental electron density corresponding to the ligand. Indeed, these maps are very well defined when the ligand is bound in exactly the same orientation in all molecules, as illustrated in fig- 
ures $2 \mathrm{~b}$ and $3 \mathrm{~d}$. More diffuse maps are obtained when the affinity is lower, because the binding pockets are not fully occupied, or when several possible orientations can be achieved by the ligand, as observed for small acetylated molecules (fig. 3b, c). Increasing the ligand concentration for CysNAc did not improve the quality of the experimental electron density maps but instead has lead to nonspecific binding to different surface areas of L-ficolin, (fig. 3a). In contrast to this apparent lack of specificity for small acetylated ligands, we observed a full set of stabilizing $\mathrm{H}$-bond interactions for a galactose molecule bound in S2 (fig. 3d). Moreover, a specific extended interaction network stabilizes 4 consecutive glucose units of 1-3- $\beta$ $\mathrm{D}$-glucan at the surface of sites S3 and S4 (fig. 2d). This latter example is interesting because L-ficolin was shown to be the only plasmatic protein able to bind $\beta$-D-glucan [9], a well known pathogen-associated molecular pattern, specifically found at the surface of fungi and also present in zymosan extracts. The extended binding interaction observed in this case strongly suggests that L-ficolin has evolved towards the specific recognition of elongated molecules, as found for example in bacterial capsular polysaccharides.

\section{Concluding Remarks}

What have we learned from these structural studies and how does this open the way to new investigations? The 3 human ficolins share a common trimeric assembly and a common use of multivalent binding avidity to surfaces. Highly specific recognition of $\mathrm{N}$-acetyl derivatives is observed in the $\mathrm{S} 1$ binding site in $\mathrm{M}$-ficolin, a property that has been conserved through evolution since it is already present in the invertebrate TL5A [26]. Despite these common properties, $\mathrm{X}$-ray structural studies have shown how the 3 human ficolins harbor distinct properties, such as ligand specificity and local flexibility. These observations provide a strong structural basis to understand how proteins with complementary functional spectra can be derived from evolution from a common ancestor.

We have seen how the homologous S1 binding site in $\mathrm{H}$-ficolin has been reshaped, hence altering its recognition spectrum. We have established the structural basis for its distinct specificity towards D-fucose, which is a rare constituent of bacterial polysaccharides, almost exclusively present in LPS O-antigens [30]. This raises the following questions. Are there other $\mathrm{H}$-ficolin physiological targets in addition to $A$. viridans? And which are the precise molecular markers recognized by $\mathrm{H}$-ficolin in the

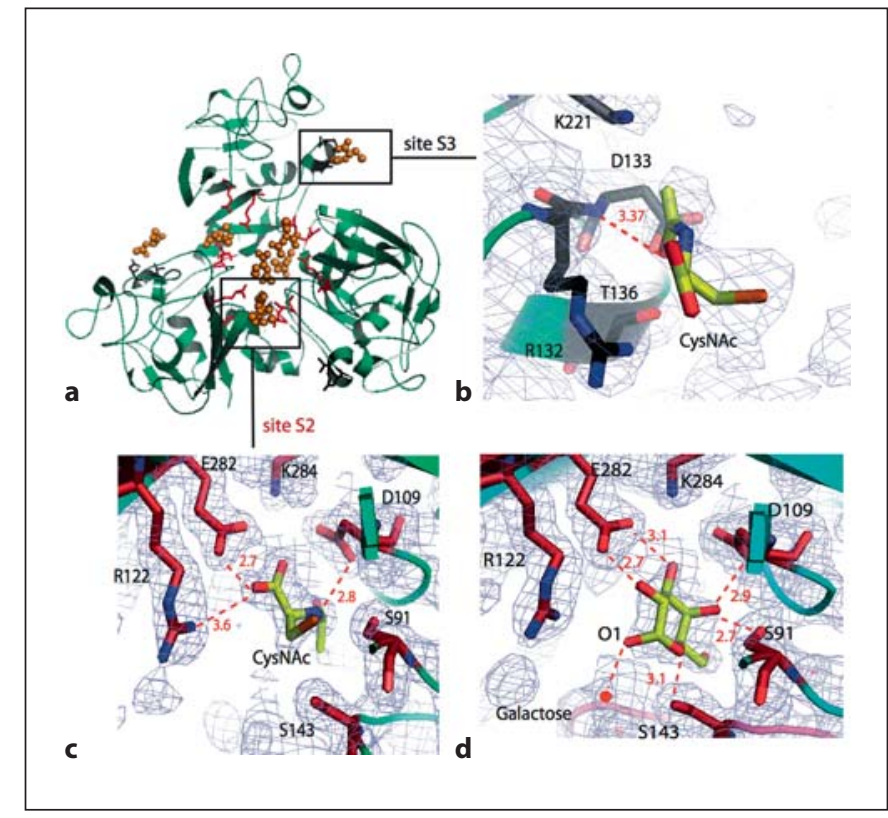

Fig. 3. Specific and non-specific ligand binding in L-ficolin. a CysNAc (orange balls) can bind to sites S2 (red) and S3 (black) of L-ficolin but it also shows nonspecific binding to several other sites when the ligand concentration is high. $\mathbf{b}$ Binding of CysNAc in site S3. c Binding of CysNac in site S2. d Highly specific binding of galactose in site S2. Polar interactions are represented by dashed red lines. A water molecule is shown as a red dot. The $2 \mathrm{Fo}-$ Fc electron density map is shown in blue.

polysaccharides of this pathogen and at the surface of apoptotic cells?

L-ficolin shows a broader recognition spectrum, which includes unrelated compounds such as neutral carbohydrates or acetylated compounds. Our studies have revealed in this protein a novel binding area comprising different sub-sites [23], a finding that reconciles previous observations. Moreover, the restricted plasticity of its inter-protomer interface may help to accommodate its ligands. Altogether, we now better understand how L-ficolin can bind specifically to several complex elongated polysaccharides (fig. 1e), such as zymosan or components of the capsules of type B Streptococci [23]. Careful interpretations of competition experiments should be performed in the case of L-ficolin since we have observed nonspecific binding of small acetylated compounds on its charged extended binding area (fig. 3a). Calcium-dependent binding to some ligands has been reported for L-ficolin $[3,7]$. However, in contrast to the known case of MBL calcium-dependent binding, the conserved calcium ions in ficolins do not interact directly with the ligands. 
Thus, the sensitivity to EDTA in some binding assays likely results from an indirect effect related to a decrease in the structural stabilization of the S1 binding site, which is close to the calcium ion. L-ficolin has been shown to bind apoptotic cells, but the precise molecular target for this binding remains to be identified. Since changes in cell surface glycoconjugates occur during apoptosis, with an increased exposition of $\beta$-D-galactose-rich plasma membrane glycoproteins [31], the newly discovered L-ficolin inner binding site, which specifically binds galactose, could be involved in this process.

Binding to sialic acids is a property restricted to human $\mathrm{M}$-ficolin and to its mouse counterpart, and we have identified a set of surface substitutions that may be structurally related to this unique property. This specificity opens new questions since numerous plasma proteins and blood cells surfaces are highly sialylated. Is this the reason why M-ficolin was initially observed at the surface of monocytes? And what is then the function of this protein?

Finally, the binding site in $\mathrm{M}$-ficolin is subject to a $\mathrm{pH}$ dependent conformational switch, being dislocated at acidic $\mathrm{pH}$. Comparison of the structures obtained at neutral and acidic $\mathrm{pH}$ has allowed the first structural insights into this unique property [24]. Does it play a physiological role in the functional cycle of $\mathrm{M}$-ficolin, including steps in acidic compartments such as phagolysosomes, where ligands could be released? Further investigations at the molecular and cellular levels should provide answers to these questions.

\section{References}

1 Medzhitov R: Recognition of microorganisms and activation of the immune response. Nature 2007;449:819-826.

2 Fujita T, Matsushita M, Endo Y: The lectincomplement pathway: its role in innate immunity and evolution. Immunol Rev 2004 198:185-202.

3 Matsushita M, Endo Y, Taira S, Sato Y, Fujita T, Ichikawa N, Nakata M, Mizuochi T: A novel human serum lectin with collagenand fibrinogen-like domains that functions as an opsonin. J Biol Chem 1996;271:24482454.

4 Sugimoto R, Yae Y, Akaiwa M, Kitajima S, Shibata Y, Sato H, Hirata J, Okochi K, Izuhara K, Hamasaki N: Cloning and characterization of the Hakata antigen, a member of the ficolin/opsonin p35 lectin family. J Biol Chem 1998;273:20721-20727.

$\checkmark 5$ Harumiya S, Takeda K, Sugiura T, Fukumoto Y, Tachikawa H, Miyazono K, Fujimoto D, Ichijo $\mathrm{H}$ : Characterization of ficolins as novel elastin-binding proteins and molecular cloning of human ficolin-1. J Biochem (Tokyo) 1996;120:745-751.

-6 Honoré C, Rørvig S, Munthe-Fog L, Hummelshøj T, Madsen HO, Borregaard N, Garred P: The innate pattern recognition molecule Ficolin-1 is secreted by monocytes/ macrophages and is circulating in human plasma. Mol Immunol 2008;45:2782-2789.

7 Krarup A, Thiel S, Hansen A, Fujita T, Jensenius JC: L-ficolin is a pattern recognition molecule specific for acetyl groups. J Biol Chem 2004;279:47513-47519.
-8 Lynch NJ, Roscher S, Hartung T, Morath S, Matsushita M, Maennel DN, Kuraya M, Fujita T, Schwaeble WJ: L-ficolin specifically binds to lipoteichoic acid, a cell wall constituent of Gram-positive bacteria, and activates the lectin pathway of complement. J Immunol 2004;172:1198-1202.

9 Ma YG, Cho MY, Zhao M, Park JW, Matsushita M, Fujita T, Lee BL: Human mannosebinding lectin and L-ficolin function as specific pattern recognition proteins in the lectin activation pathway of complement. J Biol Chem 2004;279:25307-25312.

10 Aoyagi Y, Adderson EE, Min JG, Matsushita M, Fujita T, Takahashi S, Okuwaki Y, Bohnsack JF: Role of L-ficolin/mannose-binding lectin-associated serine protease complexes in the opsonophagocytosis of type III group B Streptococci. J Immunol 2005; 174:418425.

11 Tsujimura M, Ishida C, Sagara Y, Miyazaki T, Murakami K, Shiraki H, Okochi K, Maeda Y: Detection of serum thermolabile beta-2 macroglycoprotein (Hakata antigen) by enzyme-linked immunosorbent assay using polysaccharide produced by Aerococcus viridans. Clin Diagn Lab Immunol 2001;8:454459.

12 Lacroix M, Dumestre-Pérard C, Schoehn G, Houen G, Cesbron J-Y, Arlaud GJ, Thielens NM: Residue Lys57 in the collagenous region of human L-ficolin and its counterpart Lys 47 in $\mathrm{H}$-ficolin play a key role in the interaction with the MASPs and the collectin receptor calreticulin. J Immunol 2009; 182:456-465.

13 Liu Y, Endo Y, Iwaki D, Nakata M, Matsushita M, Wada I, Inoue K, Munakata M, Fujita T: Human M-ficolin is a secretory protein that activates the lectin complement pathway. J Immunol 2005;175:3150-3156.
14 Frederiksen PD, Thiel S, Larsen CB, Jensenius JC: M-ficolin, an innate immune defence molecule, binds patterns of acetyl groups and activates complement. Scand J Immunol 2005;62:462-473.

15 Teh C, Le Y, Lee SH, Lu J: M-ficolin is expressed on monocytes and is a lectin binding to $\mathrm{N}$-acetyl-D-glucosamine and mediates monocyte adhesion and phagocytosis of Escherichia coli. Immunology 2000;101:225232.

$>16$ Matsushita M, Fujita T: Ficolins and the lectin complement pathway. Immunol Rev 2001;180:78-85

17 Thiel S, Vorup-Jensen T, Stover CM, Schwaeble W, Laursen SB, Poulsen K, Willis AC, Eggleton P, Hansen S, Holmskov U, Reid $\mathrm{KB}$, Jensenius JC: A second serine protease associated with mannan-binding lectin that activates complement. Nature 1997;386: 506-510.

18 Pagh R, Duus K, Laursen I, Hansen PR, Thielens N, Arlaud GJ, Kongerslev L, Højrup P, Houen G: The chaperone and potential mannan-binding lectin (MBL) co-receptor calreticulin interacts with $\mathrm{MBL}$ through the binding site for MBL-associated serine proteases. FEBS J 2008;275:515-526.

19 Matsushita M, Fujita T: Activation of the classical complement pathway by mannosebinding protein in association with a novel C1s-like serine protease. J Exp Med 1992; 176:1497-1502.

20 Dahl MR, Thiel S, Matsushita M, Fujita T, Willis AC, Christensen T, Vorup-Jensen T, Jensenius JC: MASP-3 and its association with distinct complexes of the mannanbinding lectin complement activation pathway. Immunity 2001;15:127-135. 
21 Stover CM, Thiel S, Thelen M, Lynch NJ, Vorup-Jensen T, Jensenius JC, Schwaeble W: Two constituents of the initiation complex of the mannan-binding lectin activation pathway of complement are encoded by a single structural gene. J Immunol 1999;162:34813490.

22 Takahashi M, Endo Y, Fujita T, Matsushita $\mathrm{M}$ : A truncated form of mannose-binding lectin-associated serine protease (MASP)-2 expressed by alternative polyadenylation is a component of the lectin complement pathway. Int Immunol 1999;11:859-863.

23 Garlatti V, Belloy N, Martin L, Lacroix M, Matsushita M, Endo Y, Fujita T, FontecillaCamps JC, Arlaud GJ, Thielens NM, Gaboriaud C: Structural insights into the innate immune recognition specificities of $\mathrm{L}$ - and H-ficolins. EMBO J 2007;26:623-633.
24 Garlatti V, Martin L, Gout E, Reiser JB, Fujita T, Arlaud GJ, Thielens NM, Gaboriaud C: Structural sensing by M-ficolins and its control by a pH-dependent conformational switch. J Biol Chem 2007;282:35814-35820.

25 Tanio M, Kondo S, Sugio S, Kohno T: Trivalent recognition unit of innate immunity system: crystal structure of trimeric human M-ficolin fibrinogen-like domain. J Biol Chem 2007;282:3889-3895.

26 Kairies N, Beisel HG, Fuentes-Prior P, Tsuda $\mathrm{R}$, Muta T, Iwanaga S, Bode W, Huber R, Kawabata SI: The 2.0-A crystal structure of tachylectin 5A provides evidence for the common origin of the innate immunity and the blood coagulation systems. Proc Natl Acad Sci USA 2001;98:13519-13524.

27 Gaboriaud C, Juanhuix J, Gruez A, Lacroix M, Darnault C, Pignol D, Verger D, Fontecilla-Camps JC, Arlaud GJ: The crystal structure of the globular head of complement protein Clq provides a basis for its versatile recognition properties. J Biol Chem 2003;278:46974-46982.
28 Sheriff S, Chang CY, Ezekowitz AB: Human mannose-binding protein carbohydrate recognition domain trimerizes through a triple a-helical coiled-coil. Nat Struct Biol 1994;1: 789-794.

29 Tanio M, Kohno T: Histidine-regulated activity of M-ficolin. Biochem J 2009;417:485491.

30 Kählig H, Kolarich D, Zayni S, Scheberl A, Kosma P, Schaffer C, Messner P: N-acetylmuramic acid as capping element of alphaD-fucose-containing S-layer glycoprotein glycans from Geobacillus tepidamans GS597T. J Biol Chem 2005;280:20292-20299.

31 Bilyy R, Stoika R: Search for novel cell surface markers of apoptotic cells. Autoimmunity 2007;40:249-253. 